\title{
APPROXIMATING RESIDUAL SETS BY STRONGLY RESIDUAL SETS ${ }^{1}$
}

\author{
D. A. MORAN
}

Abstract. Let $M$ be a closed topological manifold, $R$ residual in $M$, and $N$ any neighborhood of $R$ in $M$. The fulfillment by $R$ of a certain local separation property in $M$ implies that there exists a topological spine $R^{\prime}$ of $M$ such that $N \supset R^{\prime} \supset R$. (Topological spine =strongly residual set.) This local separation property is satisfied whenever $R$ is an ANR, or when $\operatorname{dim} R \leqq \operatorname{dim} M-2$.

Let $M$ be a closed topological $n$-manifold. Following Doyle and Hocking [2], a subset $R$ of $M$ is said to be residual in $M$ if $M-R$ is a topological open $n$-cell which is dense in $M$. In [3], such a subset was called strongly residual if it can be realized as $\phi\left(\dot{I}^{n}\right)$ for some map $\phi$ satisfying the criteria of the mapping theorem of Brown and Casler [1]. The main result contained herein represents some progress toward determining which residual sets are strongly residual: it shows that a residual set possessing a certain property akin to semi-localconnectedness can be enlarged by an arbitrarily small amount to form a set which is strongly residual. The theorem is arrived at by tampering with the proof of the Brown-Casler theorem, and only the modifications will be given here. The definitions of the terms used below are by now standard, or may be found in [1].

Theorem. Let $R$ be residual in $M$, and let $N$ be any neighborhood of $R$ in $M$. Suppose that there is a sequence $\varepsilon_{1}, \mathcal{E}_{2}, \ldots$ of finite open covers of $M$ such that (1) the diameters (relative to some fixed metric on $M$ ) of the members of $\mathcal{E}_{i}$ tend to zero as $i \rightarrow \infty$, and (2) for each element $E$ of any cover in the sequence, $E-R$ has finitely many components. Then there is a map $\phi$ from $I^{n}$ onto $M$ such that $\phi \mid I^{n}$ is a homeomorphism, $\phi^{-1} \phi\left(\dot{I}^{n}\right)$ $=\dot{I}^{n}, \operatorname{dim} \phi\left(\dot{I}^{n}\right) \leqq n-1$, and $N \supset \phi\left(\dot{I}^{n}\right) \supset R$.

Proof. By using the Lebesgue numbers of the coverings $\mathcal{E}_{i}$ and relabeling a subsequence if necessary, it can easily be shown that no generality is lost by assuming that each element of $\mathcal{E}_{i}$ is a connected

Received by the editors April 7, 1969.

AMS Subject Classifications. Primary 5478; Secondary 5460.

Key Words and Phrases. Topological manifold, residual set, strongly residual, topolngical spine, Brown-Casler map.

1 Work partially supported by NSF Grant GP-8962. 
open set of diameter less than $2^{-i-1}$, and that for each $i, \varepsilon_{i+1}$ refines $\mathcal{E}_{i}$.

Let $C_{1}$ be an $n$-cell in $M-R$ which contains a point of each component of $E-R$, for each element $E$ of $\varepsilon_{1} ; C_{1}$ can (and will) be chosen so that $M-C_{1} \subset N$, and $\dot{C}_{1}$ is bicollared. Let $X_{2}$ be a finite set of points of $M-R$ consisting of one point from each component of $E-R$, for each $E \in \mathcal{E}_{2}$. If $x \in X_{2}$, let $E_{2}$ be a member of $\mathcal{E}_{2}$ containing $x$, and suppose that $K_{2}$ is the component of $E_{2}-R$ which contains $x$. Because $K_{2}$ is connected and $\varepsilon_{2}$ refines $\varepsilon_{1}, K_{2}$ lies in a component $K_{1}$ of $E_{1}-R$, for some $E_{1} \in \mathcal{E}_{1}$.

Since $R$ is closed in $M$ and $E_{1}$ is open in $M, K_{1}$ is a component of the open subset $E_{1}-R$ of the locally-connected space $M$, hence is open in $M . K_{1}$ is contained the open $n$-cell $M-R$ and is thus open in $M-R$.

Now connectedness is equivalent to polygonal-path-connectedness for open subsets of euclidean spaces, so there is a polygonal arc (relative to some combinatorial structure on $M-R$ ) joining $x$ to some point of ${\dot{C_{1}}}_{1} \cap E_{1}$, and lying entirely within $K_{1}$.

Mutually disjoint $n$-cells containing the appropriate subarcs of the polygonal arcs thus described can now be found, and an autohomeomorphism $h_{1}$ of $M$ constructed with $h_{1}\left(C_{1}\right)$ engulfing $X_{2}$, exactly as in [1]. Repetition of the above process results in the definition of the map $\phi$, the details of the iterative process being precisely parallel to the proof in [1], the only modifications being those analogous to those described above.

Corollary. Let $R$ be residual in $M$, and let $N$ be any neighborhood of $R$ in $M$. If $\operatorname{dim} R \leqq n-2$, or if $R$ is an $A N R$, then there exists $R^{\prime}$ strongly residual in $M$ with $N \supset R^{\prime} \supset R$.

Proof. If $\operatorname{dim} R \leqq n-2$, any sequence of finite open cell covers of $M$ whose members have diameters tending to zero will satisfy the hypotheses of the theorem, since an $n$-cell cannot be separated by a set of dimension $\leqq n-2$.

If $R$ is an ANR, a sequence of covers of $M$ satisfying the hypotheses of the theorem is easily constructed by using the following

Lemma. If $X$ is an $A N R$ closed in $E^{n}, \operatorname{dim} X \leqq n-1, x \in X$, and $V$ is any neighborhood of $x$, then there exists a neighborhood $U$ of $x$ such that $U \subset V$, and $U-X$ has finitely many components.

Proof. Let $W$ be any compact neighborhood of $x$ which is contained in $V$. By the last-stated lemma in [4], $W$ meets only finitely 
many components of $V-X$. Let $K$ be the union of these components, and put $U=\operatorname{Int} \bar{K}$. $U$ clearly has the required properties.

A residual set satisfying the hypotheses of the above theorem need not itself be strongly residual, even if it has dimension $n-1$, as the following example shows:

Example. Consider $S^{3}$ as the one-point compactification of $E^{3}$, and define subsets of $S^{3}$ via a rectangular coordinate system in $E^{3}$, as follows:

$$
\begin{aligned}
& A=\{(x, y, z) \mid z=0,-1 \leqq x \leqq 0,-1 \leqq y \leqq 1\}, \\
& B=\{(x, y, z) \mid 0<x \leqq 1, y=\sin (1 / x), 0 \leqq z \leqq x\}, \\
& C=\{(x, y, z) \mid z=0,0 \leqq x \leqq \epsilon,-1 \leqq y \leqq 1\},
\end{aligned}
$$

where $\epsilon$ is any small positive number.

$A \cup B$ is not strongly residual in $S^{3}$ since, failing to be locally connected, it cannot be an ANR (see [3]). However, $A \cup B$ can easily be shown to be cellular, and this implies that it is residual in $S^{3}$. Including the additional set $C$ cleans matters up quite a bit; albeit tedious, it is by no means a herculean task to exhibit a pseudoisotopy of the cube $\{(x, y, z) \mid-1 \leqq x, y, z \leqq 1\}$ onto $A \cup B \cup C$.

The question as to whether a residual ANR can fail to be strongly residual remains open.

\section{REFERENCES}

1. M. Brown, "A mapping theorem for untriangulated manifolds," Topology of 3-manifolds and related topics (Proc. Univ. of Georgia Inst., 1961), Prentice-Hall, Englewood Cliffs, N. J. 1962, pp. 92-94. MR 28 \#1599.

2. P. H. Doyle and J. G. Hocking, A decomposition theorem for n-dimensional manifolds, Proc. Amer. Math. Soc. 13 (1962), 469-471. MR 25 \#4514.

3. D. A. Moran, A remark on the Brown-Casler mapping theorem, Proc. Amer. Math. Soc. 18 (1967), 1078. MR 36 \#7123.

4. R. H. Bing, Retractions onto ANR's, Proc. Amer. Math. Soc. 21 (1969), 618-620. MR 39 \#940.

Michigan State University, East Lansing, Michigan 48823 